\title{
Qualitative research provides insights into the experiences and perspectives of people with spinal cord injuries and those involved in their care
}

\section{R. Hall ${ }^{1}$ L. A. Harvey ${ }^{2}$}

(C) International Spinal Cord Society 2018

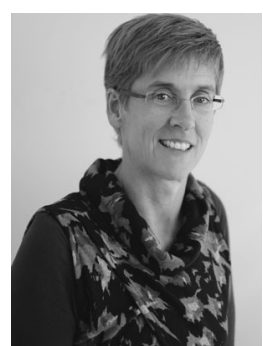

The majority of papers published in Spinal Cord use quantitative methodologies to answer a particular research question where results are largely expressed numerically. However, another whole area of research utilises qualitative methodologies. This is a broad category of research that uses a range of different methodologies and approaches to explore the experiences and perspectives of individuals, and how they make meaning of particular phenomena. For example, qualitative research could be used to explore how people with spinal cord injuries experience their care, adjust to life and prioritise their needs. Similarly it could be used to explore the perspectives of family members, carers or healthcare professionals.

Qualitative research focuses on the nuances between individuals, and aims to gain in-depth information about a specific topic from the perspective of the individual. This is generally achieved through interviews or focus groups. These allow participants to speak dynamically and extensively about a given topic. Researchers then compile a detailed analysis of each participant's account. In this context, qualitative research is important because it allows for an in-depth exploration of certain research questions that quantitative research can never answer. Needless to say, sample sizes of qualitative studies are generally small because the intention is not to ensure that the sample reflects the population, but rather to ensure the sample reflects the range and scope of perspectives and experiences of different people with varying backgrounds, opinions and conditions. This is achieved by purposive homogenous sampling.

Good quality qualitative research tends to employ some form of data triangulation during the analysis by using more than one researcher to code the data, or asking an independent peer, or participants themselves, to review coding and themes. This process can help add rigour and depth to the analysis. The idea of adding depth to the analysis is important in qualitative research as the pursuit is not just to describe what participants say, but also to capture something of what they might mean when they say certain things. For example, if participants with spinal cord injuries speak about finding access to healthcare information helpful, an experienced qualitative researcher would both describe these accounts (aided by the use of quotes), as

$\triangle$ L. A. Harvey

spinalcord@iscos.org.uk

1 Oxford Health NHS Trust, Oxford, UK

2 University of Sydney, Sydney, Australia well as provide a higher level interpretation about how participants felt the information helped them. For example, perhaps it gave them a greater sense of control or mastery over their condition.

There are a number of appraisal checklists to critique qualitative research [1-3]. Many of the items on the various checklists are similar to those used to critique quantitative research. For example the requirement that a manuscript gives a coherent account of its' rationale, aims and methods of sampling, data collection and analysis. However, many are also unique to qualitative research. These include some demonstration of reflexivity (consideration of how the researchers' decisions and beliefs may have impacted upon the research process). This is usually demonstrated via either a positioning statement about the researcher, or reference to use of bracketing interviews or reflective discussions within the research team. This is important because researchers can influence the research process through the way interview questions are framed and findings interpreted. Good quality manuscripts also publish their interview schedules and carefully describe their samples (while retaining anonymity).

This edition of Spinal Cord has a nice example of some wellconducted qualitative research from Spain [4]. The researchers explored what it means to be a relative of a person with spinal cord injury. They conducted 25 semi-structured interviews and then extracted key themes. The paper provides valuable insights into the needs and concerns of carers; people who are central to the lives of those affected by spinal cord injuries. So we encourage all to read this paper in an effort to better understand the perspective of carers and to more fully appreciate the value of well-conducted qualitative research.

\section{References}

1. Walsh D, Downe S. Appraising the quality of qualitative research. Midwifery. 2006;22:108-19.

2. Clark JP. How to peer review a qualitative manuscript. In: Godlee F, Jefferson T, (eds). Peer review in Health Sciences. 2nd edns. London: BMJ Books; 2003.

3. Yardley L. Dilemmas in qualitative research. Psychol Health. 2000;15:215-28.

4. Juguera Rodriguez L, Pardo Rios M, Leal Costa C, Castillo Hermoso M, Perez Alonso N, Diaz Agea JL. Relatives of people with spinal cord injury: a qualitative study of caregivers' metamorphosis. Spinal Cord. 2018;26:548-59. 